\title{
Seleção de doador de medula óssea ou sangue periférico
}

\section{Bone marrow or peripheral blood donor selection}

Noemi F. Pereira ${ }^{I}$

Danielli C. M. Oliveira ${ }^{2}$

Margareth Torres ${ }^{3}$

Celso A. Rodrigues ${ }^{4}$

Iracema S. B. Alencar 5

Izabella Salomão ${ }^{6}$

Marcos A. Mauad

Vergílio A. R. Colturato ${ }^{8}$

Luis Fernando S. Bouzas ${ }^{9}$

Maria Elisa Moraes $^{10}$
A compatibilidade HLA é o fator mais valorizado na escolha do doador de medula óssea voluntário, preconizando-se a realização de HLA de alta resolução nos locos HLA-A,B,C, DRB1 e DQB1. Tem sido dado preferência para o doador com consanguinidade alélica $8 \times 8(A, B, C, D R B 1)$. Na presença de incompatibilidade na classeI sugere-se a busca de doador com compatibilidade DQB1 (9x10). Já as incompatibilidades dos locos DPB1 não constituem critério de exclusão de doador, exceto quando existir presença de anticorpo contra o loco HLA-DP do doador. Rev. Bras. Hematol. Hemoter. 2010;32(Supl. 1): 3-5.

Palavras-chave: HLA de alta resolução; compatibilidade do doador.

\section{Introdução}

A seleção do doador com grau adequado de compatibilidade representa uma das estratégias essenciais para o sucesso do transplante de células-tronco hematopoéticas (TCTH). Dentre os fatores genéticos que exercem maior influência no resultado desses transplantes estão os genes do sistema HLA, caracterizados por extenso polimorfismo. O reconhecimento da ação fundamental da alogenicidade das moléculas HLA na evolução pós-transplante levou a necessidade de identificação das variantes alélicas dos genes HLA, ou de seus produtos, no paciente e nos seus potenciais doadores, e esta informação tem permitido a escolha criteriosa de doadores. A importância da compatibilidade HLA no TCTH, descrita há algumas décadas, tem sido reavaliada, nos últimos anos, a partir do desenvolvimento de novas metodologias para identificação dos genes HLA clássicos de transplantação. Diversos estudos, após análise retrospectiva dos transplantes com doadores não consanguíneos considerados idênticos para os locos HLA-A, B e DRB1, revelaram incompatibilidades alélicas nestes locos bem como divergências no nível antigênico e alélico para outros locos, como o HLA-C e DQB1, que não haviam sido previamente avaliados.

A metodologia para a identificação das variantes alélicas inclui a linfocitotoxicidade dependente de complemento que permite determinar as especificidades sorológicas. Atualmente, os métodos moleculares mais utilizados são a PCR-SSP (Polymerase Chain Reaction-Sequence Specific Primers) e a PCR-SSO (Polymerase Chain Reaction-

\footnotetext{
${ }^{1}$ Bioquímica. Chefe do Laboratório de Imunogenética do Hospital de Clínicas - Universidade Federal do Paraná - Curitiba-PR

Médica. Laboratório de Imunogenética do Instituto Nacional do Câncer (Inca), Rio de Janeiro-RJ

${ }^{3}$ Médica. Departamento de Patologia Clínica do Hospital Israelita Albert Einstein (HIAE), São Paulo-SP.

Médico. Disciplina de Hematologia e Hemoterapia da Universidade Federal de São Paulo (Unifesp) e Centro de Oncologia do Hospital Sírio Libanês, São Paulo-SP.

Médica. Coordenadora do Rereme/Redome - Instituto Nacional do Câncer (Inca) - Rio de Janeiro-RJ.

${ }^{6}$ Bióloga. Rereme/Redome - Instituto Nacional do Câncer (Inca) - Rio de Janeiro-RJ.

Médico hematologista do Hospital Amaral do Carvalho, Diretor do Hemonúcleo Regional de Jaú - Jaú-SP.

${ }^{8}$ Unidade de Transplante de Medula Óssea, Hemonúcleo Regional de Jaú, Hospital Amaral Carvalho - Jaú-SP

Pediatra/hematologista. Instituto Nacional de Câncer (Inca) - Rio de Janeiro-RJ.

JRM Investigações Imunológicas - Rio de Janeiro-RJ
}

Correspondência: Noemi Farah Pereira

Laboratório de Imunogenética

Rua Padre Camargo $280-1^{\circ}$ andar

80060-240 - Curitiba-PR - Brasil

E-mail:nfarah@hc.ufpr.br

Doi: 10.1590/S1516-84842010005000022 
Sequence Specific Oligonucleotide), os quais permitem que a tipagem possa ser realizada em dois níveis de resolução dependendo do conjunto de primers ou de sondas utilizado, respectivamente. Na tipagem de baixa resolução, somente os dois primeiros dígitos são identificados, indicando a que grupo os alelos pertencem, grupos esses que, em geral, correspondem às especificidades sorológicas: $A^{*} 03$ (A3), B*07 (B7), DRB1*03 (DR3), etc. A análise de alta resolução, que utiliza um conjunto adicional de primers (SSP) ou sondas (SSO), permite a identificação dos alelos propriamente ditos: A*0302, B*0702, DRB $1 * 0301$, etc. Contudo, o método mais adequado para a tipificação de alta resolução é o sequenciamento direto do DNA ou SBT (Sequence Based Typing). O desenvolvimento da metodologia molecular de alta resolução possibilitou a identificação dos alelos e, consequentemente, desvendou incompatibilidades não reveladas pelos métodos sorológicos ou moleculares de baixa resolução.

\section{Critérios para seleção de doador de medula óssea / sangue periférico não aparentado}

1. Realizar tipagem de alta resolução para os locos HLA-A, B, C, DRB1 e DQB1 de todos os pacientes referidos para a busca de doador não aparentado e de seus potenciais doadores porque as incompatibilidades alélicas podem ser funcionalmente relevantes.

2. Selecionar preferencialmente doador com compatibilidade alélica 8/8 (HLA-A, B, C e DRB1) devido ao efeito cumulativo ou sinergismo das incompatibilidades HLA. O número total de incompatibilidades HLA é fator de risco de falha de pega do enxerto, DECHa e mortalidade tanto em pacientes com doenças neoplásicas de risco padrão quanto em pacientes com doença avançada (alto risco), embora o efeito aditivo das incompatibilidades HLA seja mais pronunciado em pacientes considerados de baixo risco. Um estudo do NMDP demonstrou uma queda de cerca de $10 \%$ na sobrevida pós-transplante a cada incompatibilidade HLA adicional. ${ }^{1,2,3}$

3. Quando não houver doador com compatibilidade alélica 8/8 (HLA-A, B, C e DRB1) escolher preferencialmente:

- Doador com compatibilidade DQB1 (9/10) para evitar um "efeito aditivo de DQB1". Apesar dos resultados conflitantes entre os estudos que investigaram a influência de incompatibilidades DQ nos transplantes, alguns sugerem (sem significância estatística) um efeito aditivo de DQ ao avaliar o risco de mortalidade associado às incompatibilidades HLA. ${ }^{2,3,4}$

- Doador com compatibilidade DRB1 e uma incompatibilidade em um dos locos de classe I (A, B ou C). Em 1995, a incompatibilidade em DRB1 foi associada com maior risco de DECHa e de mortalidade. O estudo do International Histocompatibility Working Group em TCTH (2004) mostrou um efeito loco específico no grupo de pacientes caucasoides de baixo risco, onde uma incompatibilidade no loco $\mathrm{C}$ ou $\mathrm{B}$ aumentava o risco de mortalidade em relação aos demais locos; enquanto em pacientes japoneses um risco maior de mortalidade estava associado ao loco A. Um estudo mais recente do NMDP (caucasoides) mostrou que incompatibilidades nos locos HLA-B e C são mais bem toleradas do que em HLA-A e DRB1 (2007). Isto demonstra que estudos adicionais ainda são necessários para determinar se há e qual é o impacto diferencial de incompatibilidades nos diferentes locos HLA. Diferenças nos resultados podem ser decorrentes de: a) subgrupos amostrais (incompatibilidade em cada loco) não são de tamanho suficiente; b) as proporções de incompatibilidades permissíveis e não permissíveis podem não estar distribuídas de modo uniforme em cada subgrupo., ${ }^{3,5,6,7}$

- Doador com incompatibilidade de alelos ao invés de antígenos. As incompatibilidades de antígenos (baixa resolução), mas não de alelos (alta resolução) estão associadas com falha de pega do enxerto. Com respeito ao risco de mortalidade, as incompatibilidades alélicas e antigênicas apresentam efeitos similares, exceto para o loco HLA-C no qual a incompatibilidade alélica parece ser mais tolerada em relação à antigênica. ${ }^{2,3,8}$

- Incompatibilidades DPB1 não constituem critério de exclusão de doador, exceto quando o receptor apresentar anticorpos pré-formados contra moléculas HLA-DP expressas pelo doador. Apesar das incompatibilidades DP serem frequentes em pares de receptor/doador HLA-A, B, C, DRB1 e DQB1 idênticos, e alguns estudos sugerirem sua influência na ocorrência de DECH aguda, bem como na redução da taxa de recaída pós-transplante, a ausência de resultados consistentes em relação à sua influência na sobrevida pós-transplante indica que a compatibilidade para este loco não deve ser incluída como um critério definitivo para seleção de doador. ${ }^{3,9,10,11,12}$

- Fazer pesquisa de anticorpos anti-HLA de classes I e II no soro do receptor que encontrar somente doador com incompatibilidade HLA:

- Evitar doador incompatível em qualquer loco HLA quando o receptor apresentar anticorpos específicos para moléculas HLA expressas pelo doador.

- Quando o receptor apresentar anticorpos anti-DPB1 considerar a tipagem DPB1 do par doador/receptor.

- Se não houver outro doador disponível ou tempo para iniciar outro processo de busca, empregar medidas para a remoção dos anticorpos anti-HLA pré-formados.

\section{Nivel de evidência: IC:}

Força de recomendação: forte e nível de evidência fraca para a realização de tipagem HLA alta resolução locos A, B, C e DRB1. Os achados destes estudos são relevantes em demonstrar evidências indiretas de benefícios inequívocos (taxa de sobrevida, taxa de DECH, falha de pega, mortalidade) de maior compatiblidade HLA, em transplantes não relacionados. 


\begin{abstract}
The HLA system is considered the most important factor in choosing a volunteer bone marrow donor with the recommendation of performing high resolution HLA tests for the HLA-A, B, C, DRB1 and $D Q B 1$ loci. A preference has been given for donor $8 \times 8$ (A, $B$, $C, D R B 1)$ allele matching. In the presence of class-I incompatibility a search for DQB1 (9x10) donor compatibility is suggested. The incompatibility of the DPB1 locus does not constitute exclusion of the donor, except when there is the presence of antibodies against the HLA-DP locus of the donor. Rev. Bras. Hematol. Hemoter. 2010; 32(Supl. 1):3-5.
\end{abstract}

Key words: High resolution HLA tests; donor compatibility.

\section{Referências Bibliográficas}

1. Morishima Y, Sasazuki T, Inoko H, Juji T, Akaza T, Kodera Y, et $a l$. The clinical significance of human leukocyte antigen (HLA) allele compatibility in patients receiving a marrow transplant from serologically HLA-A, HLA-B, and HLA-DR matched uhnrelated donors. Blood. 2002;99(11):4200-06.

2. Petersdorf EW, Anasetti C, Martin PJ, Gooley T, Radich J, Malkki $\mathrm{M}$, et al. Limits of HLA mismatching in unrelated hematopoietic cell transplantation. Blood. 2004;104(9):2976-80.

3. Lee SJ, Klein J, Haagenson M, Baxter-Lowe AL, Confer LD, Eapen M, et al. High-resolution donor-recipient HLA matching contributes to the success of unrelated donor marrow transplantation. Blood. 2007;110(13):4576-83.

4. Flomenberg N, Baxter-Lowe LA, Confer D, Fernandez-Vina M, Filipovich A, Weisdorf D, et al. Impact of HLA class I and class II high-resolution matching on outcomes of unrelated donor bone marrow transplantation: HLA-C mismatching is associated with a strong adverse effect on transplantation outcome. Blood. 2004; 104(7):1923-30.

5. Petersdorf EW, Longton GM, Anasetti C, Martin PJ, Mickelson EM, Smith AG, et al. The significance of HLA-DRB1 matching on clinical outcome after HLA-A, B, DR identical unrelated donor marrow transplantation. Blood. 1995;86(4):1606-13.

6. Petersdorf EW, Gooley T, Malkki M, Horowitz M. Clinical significance of donor-recipient HLA matching on survival after myeloablative hematopoietic cell transplantation from unrelated donors tissue antigens. Tissue Antigens. 2007; 69 (suppl 1):25-30.

7. Morishima Y, Kawase T, Malkki M. Effect of HLA-A2 allele disparity on clinical outcome in hematopoietic cell transplantation from unrelated donors. Tissue Antigens. 2007; 69(supp1 1):31-5.

8. Petersdorf EW, Hansen JA, Martin PJ, Woolfrey A, Malkki M, Gooley T, et al. Major-histocompatibility-complex class I alleles and antigens in hematopoietic-cell transplantation. New Engl J Med. 2001;345(25):1794-1800.

9. Petersdorf WE, Smith AG, Mickelson EM, Longton GM, Anasetti C, Choo SY, et al. The role of HLA-DPB1 disparity in the development of acute graft-versus host disease following unrelated donor marrow transplantation. Blood. 1993;81(7):1923-32.

10. Petersdorf EW, Gooley T, Malkki M, Anasetti C, Martin P, Woolfrey A, et al. The biological signicance of HLA-DP gene variation in haematopoietic cell transplantation. Br J Haematol. 2001;112(4):988-94.
11. Shaw BE, Marsh SG, Mayor NP, Russell NH, Madrigal JA. HLADPB1 matching status has significant implications for recipients of unrelated donor stem cell transplants. Blood. 2006; 107 (3):1220-26.

12. Shaw BE, Gooley TA, Malkki M, Madrigal JA, Begovich AB, Horowitz MM, et al. The importance of HLA-DPB1 in unrelated donor hematopoietic cell transplantation. Blood 2007; 110(13): 4560-66.

13. Anasetti C, Amos D, Beatty PG, Appelbaum FR, Bensinger W, Buckner CD, et al. Effect of HLA compatibility on engraftment of bone marrow transplants in patients with leukemia or lymphoma. N Engl J Med 1989;320(4):197-204.

Este artigo foi avaliado pelos coordenadores das Diretrizes do Transplante de Medula Óssea da Sociedade Brasileira de Transplante de Medula Óssea, Luis Fernando Bouzas, Prof. Julio Cesar Voltarelli e Nelson Hamerschlak, e publicado após avaliação e revisão do editor, Milton Artur Ruiz.

Conflito de interesse: sem conflito de interesse

Recebido: 09/11/2009

Aceito: 23/11/2009 